\title{
HOW DO RARE EARTH ELEMENTS (LANTHANOIDS) AFFECT ROOT DEVELOPMENT AND PROTOCORM-LIKE BODY FORMATION IN HYBRID CYMBIDIUM?
}

\author{
Jaime A. TEIXEIRA DA SILVA ${ }^{1,2}$ \\ ${ }^{1}$ Faculty of Agriculture and Graduate School of Agriculture, Kagawa University, \\ Miki cho, Kita Gun, Ikenobe, 761-0795, Japan \\ ${ }^{2}$ Current address: P. O. Box 7, Ikenobe 3011-2, Miki-cho, Kita-gun, Kagawa, 761-0799, Japan
}

Received: January 9, 2014; Accepted: March 20, 2014

\begin{abstract}
Only few studies in the plant tissue culture literature have examined the impact of lanthanoids, or rare earth elements, on in vitro plant organogenesis. In this study, using a model plant, hybrid Cymbidium Twilight Moon 'Day Light', the impact of six lanthanoids (lanthanum (III) nitrate hexahydrate $\left(\mathrm{La}\left(\mathrm{NO}_{3}\right)_{3} \cdot 6 \mathrm{H}_{2} \mathrm{O}\right)$, cerium (III) nitrate hexahydrate $\left(\mathrm{Ce}\left(\mathrm{NO}_{3}\right)_{3} \cdot 6 \mathrm{H}_{2} \mathrm{O}\right)$, neodymium (III) nitrate hexahydrate $\left(\mathrm{Nd}\left(\mathrm{NO}_{3}\right)_{3} \cdot 6 \mathrm{H}_{2} \mathrm{O}\right)$, praseodymium (III) nitrate hexahydrate $\left(\operatorname{Pr}\left(\mathrm{NO}_{3}\right)_{3} \cdot 6 \mathrm{H}_{2} \mathrm{O}\right)$, samarium (III) nitrate hexahydrate $\left(\mathrm{Sm}\left(\mathrm{NO}_{3}\right)_{3} \cdot 6 \mathrm{H}_{2} \mathrm{O}\right)$, gadolinium (III) nitrate hexahydrate $\left(\mathrm{Gd}\left(\mathrm{NO}_{3}\right)_{3} \cdot 6 \mathrm{H}_{2} \mathrm{O}\right)$ on new protocorm-like body (neo-PLB) formation on Teixeira Cymbidium (TC) medium was examined. 0 (control), 1, 2, 4 and $8 \mathrm{mg} \cdot \mathrm{dm}^{-3}$ of each lanthanoid was tested. All lanthanoids could produce more neo-PLBs and neo-PLB fresh weight than TC medium lacking plant growth regulators (PGRs), suggesting some PGR-like ability of lanthanoids, although PLB-related traits (percentage of half-PLBs forming neo-PLBs; number of neo-PLBs formed per half-PLB; fresh weight of half-PLB + neo-PLBs) was always significantly lower than TC medium containing PGRs. Except for Gd, all other lanthanoids had no negative impact on the number of new leaves from neo-PLB-derived shoots, but all lanthanoids showed a significantly lower plant height, shoot fresh weight and shoot dry weight and, in most cases, SPAD (chlorophyll content) value. In addition, using the same concentration of the six lanthanoids, the ability to fortify root formation of neo-PLB-derived plantlets was also assessed. Except for Sm, all other lanthanoids significantly increased the number of roots, root fresh and dry weight.
\end{abstract}

Key words: lanthanoid, orchid, PLB, rare earth element, root formation, Teixeira Cymbidium (TC) medium

\section{INTRODUCTION}

In vitro regeneration protocols for the most common orchid genera such as Paphiopedilum, Dendrobium, Phalaenopsis, Vanda, Oncidium, Epidendrum and some other orchids have been well developed (Hossain et al. 2013). In almost all cases, the use of plant growth regulators (PGRs) has been used to induce protocorm-like bodies (PLBs). These PLBs, in general, when maintained on the same medium, within 3-6 months, depending on the orchid genus, spuriously form shoots from the apical terminal of the PLB and roots from the basal portion, developing plantlets within 6-12 months, also depending on the genus or even cultivar. PGRs are the most commonly used growth-regulating substance used in orchid biotechnology, although substances such as phloroglucinol or coconut water have growth-promoting and growth-inhibiting properties, also depending on the concentration applied to medium (Teixeira da Silva 2013a; Teixeira da Silva et al. 2013). In this study, hybrid Cymbidium (Orchidaceae) has been used because its developmental response in vitro using its clonal propagule, the PLB, equivalent to a somatic embryo, has been well studied (Teixeira da Silva 
\& Tanaka 2006; Teixeira da Silva 2013b; Teixeira da Silva \& Dobránszki 2013). PLBs, when encapsulated, can form synthetic seeds (synseed) (Teixeira da Silva 2012a), which are useful units for cryopreservation (Sharma et al. 2013).

Rare earth elements (REEs) or lanthanoids are a group of 15 metallic chemical elements with atomic numbers 57-76 (Wikipedia 2013). Only few studies in the literature exist on the effect of lanthanoids on in vitro plant growth, one of them, interestingly enough, on another orchid, Dendrobium densiflorum (Luo et al. 2008). In that study, lanthanoids were shown to improve rooting. Improved rooting through the application of lanthanoids has already been shown for several crops. Lanthanum (La) and/or cerium (Ce), significantly elongated roots in Zea mays (corn) and Vigna radiata L.R. Wilczek (mungbean) or flowering in Arabidopsis thaliana (Diatloff et al. 1995a, b; He \& Loh 2000; Liu \& Hasenstein 2005). However, these studies examined the effects of lanthanoids in planta and in an ex vitro environment and thus have limited application for in vitro studies.

On the basis of the Luo et al. (2008) study, and departing from the premise that lanthanoids would have some similar effect on other orchids, a wider range of lanthanoids, six in total, was tested to assess the impact on hybrid Cymbidium organogenesis. Many media can support the induction and development of Cymbidium PLBs in vitro (Teixeira da Silva et al. 2005), Teixeira Cymbidium (TC) No. 1 medium (Teixeira da Silva 2012b) was used in this study.

\section{MATERIALS AND METHODS}

All protocols (experimental design, chemicals, reagents, explant preparation and treatment analysis) strictly follow Teixeira da Silva (2013c), almost verbatim in parts.

\section{Chemicals and reagents}

All chemicals and reagents were of the highest analytical grade available and were purchased from either Sigma-Aldrich (St. Louis, USA), Wako Chemical Co. (Osaka, Japan) or Nacalai Tesque (Kyoto, Japan), the cheapest choice at the highest tissue-culture grade, unless specified otherwise.

\section{Plant material and culture conditions}

PLBs of hybrid Cymbidium Twilight Moon 'Day Light' (Bio-U, Tokushima, Japan) originally developed from shoot-tip culture on Vacin \& Went (1949) (VW) agar medium without PGRs, were induced and subcultured (PLB induction and proliferation medium) every two months on TC medium (Teixeira da Silva 2012b), which contains a unique composition of macro- and micronutrients, and was supplemented with $0.1 \mathrm{mg} \cdot \mathrm{dm}^{-3} \alpha$-naphthaleneacetic acid (NAA) and $0.1 \mathrm{mg} \cdot \mathrm{dm}^{-3}$ kinetin (Kin), $2 \mathrm{~g} \cdot \mathrm{dm}^{-3}$ tryptone and $20 \mathrm{~g} \cdot \mathrm{dm}^{-3}$ sucrose and solidified with $8 \mathrm{~g} \cdot \mathrm{dm}^{-3}$ Bacto agar (Difco Labs., USA), following Teixeira da Silva et al. (2005) and Teixeira da Silva $\&$ Tanaka (2006). All media were adjusted to $\mathrm{pH} 5.3$ with $1 \mathrm{~N} \mathrm{NaOH}$ or $\mathrm{HCl}$ prior to autoclaving at $100 \mathrm{KPa}$ for $17 \mathrm{~min}$. Cultures were kept on $40 \mathrm{ml}$ medium in $100 \mathrm{ml}$ Erlenmeyer flasks, double-capped with aluminium foil, at $25^{\circ} \mathrm{C}$, under a $16 \mathrm{~h}$ photoperiod with a light intensity of $45 \mu \mathrm{mol} \cdot \mathrm{m}^{-2} \cdot \mathrm{s}^{-1}$ provided by $40 \mathrm{~W}$ plant growth fluorescent lamps (Homo Lux, Matsushita Electric Industrial Co., Japan). Longitudinally dissected as two pieces of PLB (3-4 $\mathrm{mm}$ in diameter) segments, 10/flask, were used as explants for PLB induction and proliferation. Culture conditions and media followed the recommendations previously established for medium formulation (Teixeira da Silva et al. 2005), biotic (Teixeira da Silva et al. 2006b) and abiotic factors (Teixeira da Silva et al. 2006a) for PLB induction, formation and proliferation.

\section{Response of Cymbidium to lanthanoids}

The effect of six lanthanoids (lanthanum (III) nitrate hexahydrate $\left(\mathrm{La}\left(\mathrm{NO}_{3}\right)_{3} \cdot 6 \mathrm{H}_{2} \mathrm{O}\right)$, cerium (III) nitrate hexahydrate $\left(\mathrm{Ce}\left(\mathrm{NO}_{3}\right)_{3} \cdot 6 \mathrm{H}_{2} \mathrm{O}\right)$, neodymium (III) nitrate hexahydrate $\left(\mathrm{Nd}\left(\mathrm{NO}_{3}\right)_{3} \cdot 6 \mathrm{H}_{2} \mathrm{O}\right)$, praseodymium (III) nitrate hexahydrate $\left(\operatorname{Pr}\left(\mathrm{NO}_{3}\right)_{3} \cdot 6 \mathrm{H}_{2} \mathrm{O}\right)$, samarium (III) nitrate hexahydrate $\left(\mathrm{Sm}\left(\mathrm{NO}_{3}\right)_{3} \cdot 6 \mathrm{H}_{2} \mathrm{O}\right)$, gadolinium (III) nitrate hexahydrate $\left(\mathrm{Gd}\left(\mathrm{NO}_{3}\right)_{3} \cdot 6 \mathrm{H}_{2} \mathrm{O}\right)$ on neo-PLB induction from halfPLBs was assessed by adding 0 (control), 1, 2, 4 and $8 \mathrm{mg} \cdot \mathrm{dm}^{-3}$ of each lanthanoid to solid or liquid TC medium (without PGRs) using the experimental design of Teixeira da Silva (2012b). The logic behind removing PGRs was to assess whether lanthanoids could effectively induce PLBs or roots in the absence of PGRs and thus test their PGR-like ability. 
All lanthanoids were dissolved in distilled water and needed concentrations were made up from 100x stock solutions fresh for each repetition. Lanthanoids were only added to solid or liquid TC medium after cooling at room temperature and filtering through $22 \mu \mathrm{m}$ Millipore filters.

Using the protocol of Teixeira da Silva et al. (2007) for the acclimatisation of Cymbidium to ex vitro conditions, shoots $4 \mathrm{~cm}$ long containing three ensheathed leaves were placed in PGR-free TC medium solidified with $2 \mathrm{~g} \cdot \mathrm{dm}^{-3}$ Gellan gum, but containing 0 (control), 1, 2, 4 and $8 \mathrm{mg} \cdot \mathrm{dm}^{-3}$ of each lanthanoid listed above. Plantlet growth was quantified by the number of new leaves and roots, plant height, fresh and dry weight of shoots and roots. Chlorophyll content in the third fully developed leaf (counting downward from the top, except for IPM in Table 2) of the plantlets was measured as the SPAD value by a chlorophyll metre (SPAD-502, Minolta, Japan).

Finally, and simultaneously to the second experiment, using the experimental design of Teixeira da Silva (2013c), $5 \mathrm{~mm}$ long roots with intact root tips were excised with a feather blade from 6 month-old shoots. These shoots were derived from control neo-PLBs derived from standard TC medium. Roots were grown on PGR-free TC medium solidified with $2 \mathrm{~g} \cdot \mathrm{dm}^{-3}$ Gellan gum (see figures in Teixeira da Silva 2013c). Using excised root tips (5 mm long), the effect of lanthanoids on root growth (as isolated organs as opposed to organs attached to the rest of the plant) was assessed. Growth of roots on solid and liquid medium was assessed after 60 days. Liquid medium was agar- and PGRfree liquid TC medium containing the same concentration of lanthanoids as the solid medium trials. Ten roots with intact root tips were cultured in $25 \mathrm{ml}$ of this medium in $250 \mathrm{ml}$ Erlenmeyer flasks and placed on a shaker at constant $84 \mathrm{rpm}$ under the same light and temperature conditions as solid TC medium. The resulting organogenic outcome (neoPLB or root response) was scored visually after
60 days, 60 days being the optimal time for sampling (Teixeira da Silva \& Dobránszki 2013). Explants and roots were photographed using stereo light microscopy and/or a digital camera.

\section{Statistical analyses}

Experiments were organised according to a randomised complete block design (RCBD) with 10 replicates per treatment (i.e., lanthanoid concentration). All experiments were repeated in triplicate $(n=30$, total sample size per treatment i.e., over three blocks). Data was subjected to analysis of variance (ANOVA) with mean separation by Duncan's multiple range test (DMRT) using SAS $^{\circledR}$ version 6.12 (SAS Institute, Cary, NC, USA). Significant differences between means were assumed at $\mathrm{p} \leq 0.05$.

\section{RESULTS AND DISCUSSION}

The most notable (original) finding of this paper is that all six lanthanoids at all concentrations reduced neo-PLB formation and all three neo-PLBrelated parameters (Table 1; Fig. 1B-H). Except for $\mathrm{Gd}$, all other lanthanoids reduced shoot-related parameters (Table 2) and stimulated root growth, development and formation, as exemplified by significantly higher number of roots, root fresh and dry weight (Table 3). On a fresh and dry weight basis, most treatments with lanthanoids, and both controls showed a root: shoot ratio of $<1.0$, indicating that the formation of shoots was favoured (Table 3 ). In the control, shoots were strongly favoured. In several instances, on a dry weight basis, root formation was strongly favoured or enhanced, namely with $4 \mathrm{mg} \cdot \mathrm{dm}^{-3} \mathrm{La}, 1$ or $2 \mathrm{mg} \cdot \mathrm{dm}^{-3} \mathrm{Ce}, \mathrm{Nd}$ or Pr (Table 3). This was strengthened by an increase in root tip biomass (Fig. 1A) when grown in the presence of all lanthanoids relative to both controls (data not shown). This study indicates that lanthanoids could be considered as a new class of PGR, and hence stimulate pure and applied research, although their high cost would likely make their use prohibitive to many researchers. 
Table 1. The growth and developmental response of hybrid Cymbidium Twilight Moon 'Day Light' half-PLBs to different lanthanoids at different concentrations after 60 days in culture

\begin{tabular}{|c|c|c|c|}
\hline $\begin{array}{l}\text { Medium lanthanoids } \\
\text { in } \mathrm{mg} \cdot \mathrm{dm}^{-3}\end{array}$ & $\begin{array}{c}\text { Percentage } \\
\text { of half-PLBs forming } \\
\text { neo-PLBs }(\%)^{*}\end{array}$ & $\begin{array}{l}\text { Number of neo-PLBs } \\
\text { formed per half-PLB }\end{array}$ & $\begin{array}{l}\text { Fresh weight }(\mathrm{mg}) \text { of } \\
\text { half-PLB }+ \text { neo-PLBs }\end{array}$ \\
\hline TC + PGRs + no lanthanoids 1 & $100 \mathrm{a}$ & $8.3 \mathrm{a}$ & $526 \mathrm{a}$ \\
\hline TC - PGRs + no lanthanoids ${ }^{2}$ & $26 \mathrm{de}$ & $1.6 \mathrm{de}$ & $146 \mathrm{f}$ \\
\hline $\mathrm{TC}-\mathrm{PGRs}+1 \mathrm{La}$ & $51 \mathrm{bc}$ & $4.7 \mathrm{c}$ & $381 \mathrm{bc}$ \\
\hline $\mathrm{TC}-\mathrm{PGRs}+2 \mathrm{La}$ & $64 \mathrm{~b}$ & $5.1 \mathrm{bc}$ & $367 \mathrm{c}$ \\
\hline $\mathrm{TC}-\mathrm{PGRs}+4 \mathrm{La}$ & $23 \mathrm{~cd}$ & $1.2 \mathrm{de}$ & $106 \mathrm{~g}$ \\
\hline $\mathrm{TC}-\mathrm{PGRs}+8 \mathrm{La}$ & $6 \mathrm{ef}$ & $0.3 \mathrm{e}$ & $38 \mathrm{~h}$ \\
\hline $\mathrm{TC}-\mathrm{PGRs}+1 \mathrm{Ce}$ & $55 \mathrm{bc}$ & $4.9 \mathrm{c}$ & $396 \mathrm{bc}$ \\
\hline $\mathrm{TC}-\mathrm{PGRs}+2 \mathrm{Ce}$ & $41 \mathrm{c}$ & $4.6 \mathrm{c}$ & $361 \mathrm{c}$ \\
\hline $\mathrm{TC}-\mathrm{PGRs}+4 \mathrm{Ce}$ & $16 \mathrm{e}$ & $0.6 \mathrm{e}$ & $44 \mathrm{~h}$ \\
\hline $\mathrm{TC}-\mathrm{PGRs}+8 \mathrm{Ce}$ & $0 \mathrm{f}$ & $0 \mathrm{e}$ & $0 \mathrm{i}$ \\
\hline $\mathrm{TC}-\mathrm{PGRs}+1 \mathrm{Nd}$ & $66 \mathrm{~b}$ & $6.1 \mathrm{~b}$ & $408 \mathrm{bc}$ \\
\hline $\mathrm{TC}-\mathrm{PGRs}+2 \mathrm{Nd}$ & $48 \mathrm{c}$ & $4.1 \mathrm{c}$ & $327 \mathrm{~d}$ \\
\hline $\mathrm{TC}-\mathrm{PGRs}+4 \mathrm{Nd}$ & $12 \mathrm{e}$ & $0.6 \mathrm{e}$ & $51 \mathrm{~h}$ \\
\hline $\mathrm{TC}-\mathrm{PGRs}+8 \mathrm{Nd}$ & $0 \mathrm{f}$ & $0 \mathrm{e}$ & $0 \mathrm{i}$ \\
\hline TC - PGRs + 1 Pr & $59 \mathrm{bc}$ & $5.8 \mathrm{bc}$ & $406 \mathrm{bc}$ \\
\hline TC - PGRs +2 Pr & $32 \mathrm{~d}$ & $2.3 \mathrm{~d}$ & $174 \mathrm{ef}$ \\
\hline $\mathrm{TC}-\mathrm{PGRs}+4 \mathrm{Pr}$ & $7 \mathrm{ef}$ & $0.1 \mathrm{e}$ & $28 \mathrm{hi}$ \\
\hline TC - PGRs +8 Pr & $0 \mathrm{f}$ & $0 \mathrm{e}$ & $0 \mathrm{i}$ \\
\hline $\mathrm{TC}-\mathrm{PGRs}+1 \mathrm{Sm}$ & $32 \mathrm{~d}$ & $2.8 \mathrm{~d}$ & $203 \mathrm{e}$ \\
\hline TC - PGRs + 2 Sm & $14 \mathrm{e}$ & $0.4 \mathrm{e}$ & $56 \mathrm{~h}$ \\
\hline $\mathrm{TC}-\mathrm{PGRs}+4 \mathrm{Sm}$ & $0 \mathrm{f}$ & $0 \mathrm{e}$ & $0 \mathrm{i}$ \\
\hline TC - PGRs +8 Sm & $0 \mathrm{f}$ & $0 \mathrm{e}$ & $0 \mathrm{i}$ \\
\hline TC - PGRs + 1 Gd & $69 \mathrm{~b}$ & $6.6 \mathrm{~b}$ & $436 \mathrm{~b}$ \\
\hline $\mathrm{TC}-\mathrm{PGRs}+2 \mathrm{Gd}$ & $48 \mathrm{c}$ & $4.6 \mathrm{c}$ & $373 c$ \\
\hline $\mathrm{TC}-\mathrm{PGRs}+4 \mathrm{Gd}$ & $16 \mathrm{e}$ & $0.8 \mathrm{e}$ & $62 \mathrm{~h}$ \\
\hline TC - PGRs + 8 Gd & $0 \mathrm{f}$ & $0 \mathrm{e}$ & $0 \mathrm{i}$ \\
\hline
\end{tabular}

PLB = protocorm-like body; SON = sonication; TC = Teixeira Cymbidium medium No. 1 (Teixeira da Silva 2012b). Lanthanoid abbreviations: $\mathrm{La}=$ lanthanum (III) nitrate hexahydrate $\left(\mathrm{La}\left(\mathrm{NO}_{3}\right)_{3} \cdot 6 \mathrm{H}_{2} \mathrm{O}\right) ; \mathrm{Ce}=$ cerium (III) nitrate hexa-

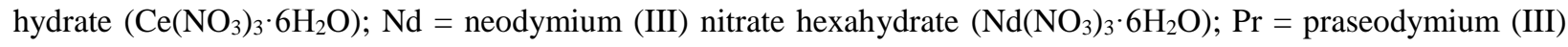
nitrate hexahydrate $\left(\mathrm{Pr}\left(\mathrm{NO}_{3}\right)_{3} \cdot 6 \mathrm{H}_{2} \mathrm{O}\right) ; \mathrm{Sm}=$ samarium (III) nitrate hexahydrate $\left(\mathrm{Sm}\left(\mathrm{NO}_{3}\right)_{3} \cdot 6 \mathrm{H}_{2} \mathrm{O}\right) ; \mathrm{Gd}=$ gadolinium (III) nitrate hexahydrate $\left(\mathrm{Gd}\left(\mathrm{NO}_{3}\right)_{3} \cdot 6 \mathrm{H}_{2} \mathrm{O}\right)$

*All percentage data was arc-sine transformed prior to analysis

Mean values followed by the same letter in the same column are not significantly different based on DMRT $(\mathrm{p}=0.05)$. $\mathrm{n}=90(10 \times 3 \times 3)$. Data was untransformed.

${ }^{1}$ Control with no lanthanoids, but including TC PGRs: $0.1 \mathrm{mg} \cdot \mathrm{dm}^{-3} \mathrm{NAA}+0.1 \mathrm{mg} \cdot \mathrm{dm}^{-3} \mathrm{Kin}$.

${ }^{2}$ Control with no lanthanoids and excluding PGRs.

${ }^{3}$ In fact, the average fresh weight of initial half-PLB explants is $54 \mathrm{mg}(\mathrm{n}=10)$. 
Table 2. Shoot (derived from neo-PLBs) growth and development of hybrid Cymbidium Twilight Moon 'Day Light' in response to different lanthanoids at different concentrations 60 days in culture after transfer to PGR-free TC medium solidified with $2 \mathrm{~g} \cdot \mathrm{dm}^{-3}$ Gellan gum

\begin{tabular}{|c|c|c|c|c|c|}
\hline $\begin{array}{l}\text { Medium lanthanoids } \\
\text { in } \mathrm{mg} \cdot \mathrm{dm}^{-3}\end{array}$ & $\begin{array}{l}\text { Number of } \\
\text { new leaves }\end{array}$ & $\begin{array}{l}\text { Plant height } \\
\qquad(\mathrm{mm})^{4}\end{array}$ & $\begin{array}{c}\text { Shoot fresh } \\
\text { weight } \\
(\mathrm{mg})^{5}\end{array}$ & $\begin{array}{c}\text { Shoot dry } \\
\text { weight }(\mathrm{mg})^{6}\end{array}$ & $\begin{array}{l}\text { SPAD } \\
\text { value }^{7}\end{array}$ \\
\hline $\mathrm{TC}+$ PGRs + no lanthanoids ${ }^{1}$ & $3.5 \mathrm{a}$ & $6.8 \mathrm{a}$ & $1294 \mathrm{a}$ & $206 \mathrm{a}$ & $44.3 \mathrm{a}$ \\
\hline TC - PGRs + no lanthanoids ${ }^{2}$ & $1.1 \mathrm{c}$ & $2.4 \mathrm{~cd}$ & $463 \mathrm{~h}$ & $56 \mathrm{~d}$ & $36.2 \mathrm{~b}$ \\
\hline $\mathrm{TC}-\mathrm{PGRs}+1 \mathrm{La}$ & $3.6 \mathrm{a}$ & $4.6 \mathrm{~b}$ & $1083 \mathrm{~b}$ & $162 \mathrm{~b}$ & $40.8 \mathrm{ab}$ \\
\hline $\mathrm{TC}-\mathrm{PGRs}+2 \mathrm{La}$ & $3.2 \mathrm{a}$ & $4.2 \mathrm{bc}$ & $962 \mathrm{c}$ & $88 \mathrm{c}$ & $38.6 \mathrm{ab}$ \\
\hline $\mathrm{TC}-\mathrm{PGRs}+4 \mathrm{La}$ & $2.2 \mathrm{~b}$ & $3.1 \mathrm{c}$ & $704 \mathrm{e}$ & $63 \mathrm{~cd}$ & $34.8 \mathrm{~b}$ \\
\hline $\mathrm{TC}-\mathrm{PGRs}+8 \mathrm{La}$ & IPM & IPM & IPM & IPM & $31.6 \mathrm{c}$ \\
\hline TC - PGRs + $1 \mathrm{Ce}$ & $3.8 \mathrm{a}$ & $4.4 \mathrm{~b}$ & $951 \mathrm{c}$ & $91 \mathrm{c}$ & $39.6 \mathrm{ab}$ \\
\hline $\mathrm{TC}-\mathrm{PGRs}+2 \mathrm{Ce}$ & $3.3 \mathrm{a}$ & $3.6 \mathrm{bc}$ & $693 \mathrm{e}$ & $71 \mathrm{~cd}$ & $34.6 \mathrm{bc}$ \\
\hline $\mathrm{TC}-\mathrm{PGRs}+4 \mathrm{Ce}$ & IPM & IPM & IPM & IPM & $32.1 \mathrm{bc}$ \\
\hline $\mathrm{TC}-\mathrm{PGRs}+8 \mathrm{Ce}$ & IPM & IPM & IPM & IPM & $30.3 \mathrm{c}$ \\
\hline $\mathrm{TC}-\mathrm{PGRs}+1 \mathrm{Nd}$ & $3.4 \mathrm{a}$ & $3.9 \mathrm{bc}$ & $801 \mathrm{~d}$ & $73 \mathrm{~cd}$ & $38.4 \mathrm{ab}$ \\
\hline $\mathrm{TC}-\mathrm{PGRs}+2 \mathrm{Nd}$ & $2.9 \mathrm{a}$ & $3.1 \mathrm{c}$ & 644 ef & $56 \mathrm{~d}$ & $34.0 \mathrm{bc}$ \\
\hline $\mathrm{TC}-\mathrm{PGRs}+4 \mathrm{Nd}$ & IPM & IPM & IPM & IPM & $30.4 \mathrm{c}$ \\
\hline $\mathrm{TC}-\mathrm{PGRs}+8 \mathrm{Nd}$ & IPM & IPM & IPM & IPM & $30.1 \mathrm{c}$ \\
\hline TC - PGRs + 1 Pr & $3.1 \mathrm{a}$ & $4.1 \mathrm{bc}$ & $816 \mathrm{~d}$ & $78 \mathrm{~cd}$ & $40.4 \mathrm{ab}$ \\
\hline TC - PGRs $+2 \mathrm{Pr}$ & $3.3 \mathrm{a}$ & $2.9 \mathrm{c}$ & $607 \mathrm{f}$ & $51 \mathrm{~d}$ & $37.3 \mathrm{~b}$ \\
\hline $\mathrm{TC}-\mathrm{PGRs}+4 \mathrm{Pr}$ & IPM & IPM & IPM & IPM & $33.6 \mathrm{bc}$ \\
\hline TC - PGRs $+8 \mathrm{Pr}$ & IPM & IPM & IPM & IPM & $31.1 \mathrm{c}$ \\
\hline TC - PGRs + $1 \mathrm{Sm}$ & $1.8 \mathrm{~b}$ & $2.6 \mathrm{~cd}$ & $603 \mathrm{f}$ & $60 \mathrm{~cd}$ & $36.1 \mathrm{~b}$ \\
\hline $\mathrm{TC}-\mathrm{PGRs}+2 \mathrm{Sm}$ & $1.2 \mathrm{c}$ & $2.1 \mathrm{~d}$ & $529 \mathrm{~g}$ & $48 \mathrm{~d}$ & $33.8 \mathrm{bc}$ \\
\hline $\mathrm{TC}-\mathrm{PGRs}+4 \mathrm{Sm}$ & IPM & IPM & IPM & IPM & $31.4 \mathrm{c}$ \\
\hline TC - PGRs $+8 \mathrm{Sm}$ & IPM & IPM & IPM & IPM & $29.6 \mathrm{c}$ \\
\hline $\mathrm{TC}-\mathrm{PGRs}+1 \mathrm{Gd}$ & $3.2 \mathrm{a}$ & $4.9 \mathrm{~b}$ & $1118 \mathrm{ab}$ & $179 \mathrm{ab}$ & $42.3 \mathrm{a}$ \\
\hline $\mathrm{TC}-\mathrm{PGRs}+2 \mathrm{Gd}$ & $2.8 \mathrm{a}$ & $4.1 \mathrm{bc}$ & $956 \mathrm{c}$ & $83 \mathrm{~cd}$ & $39.1 \mathrm{ab}$ \\
\hline TC - PGRs + 4 Gd & IPM & IPM & IPM & IPM & $38.7 \mathrm{ab}$ \\
\hline $\mathrm{TC}-\mathrm{PGRs}+8 \mathrm{Gd}$ & IPM & IPM & IPM & IPM & $34.3 \mathrm{bc}$ \\
\hline
\end{tabular}

IPM = insufficient plant material (i.e., not enough shoots from neo-PLBs); PLB = protocorm-like body; SON = sonication; TC $=$ Teixeira Cymbidium medium No. 1 (Teixeira da Silva 2012b). Lanthanoid abbreviations: La = lanthanum (III) nitrate hexahydrate $\left(\mathrm{La}\left(\mathrm{NO}_{3}\right)_{3} \cdot 6 \mathrm{H}_{2} \mathrm{O}\right) ; \mathrm{Ce}=$ cerium (III) nitrate hexahydrate $\left(\mathrm{Ce}\left(\mathrm{NO}_{3}\right)_{3} \cdot 6 \mathrm{H}_{2} \mathrm{O}\right) ; \mathrm{Nd}=$ neodymium (III) nitrate hexahydrate $\left(\mathrm{Nd}\left(\mathrm{NO}_{3}\right)_{3} \cdot 6 \mathrm{H}_{2} \mathrm{O}\right) ; \mathrm{Pr}=$ praseodymium (III) nitrate hexahydrate $\left(\mathrm{Pr}\left(\mathrm{NO}_{3}\right)_{3} \cdot 6 \mathrm{H}_{2} \mathrm{O}\right) ; \mathrm{Sm}=\mathrm{sa}-$ marium (III) nitrate hexahydrate $\left(\mathrm{Sm}\left(\mathrm{NO}_{3}\right)_{3} \cdot 6 \mathrm{H}_{2} \mathrm{O}\right)$; $\mathrm{Gd}=$ gadolinium (III) nitrate hexahydrate $\left(\mathrm{Gd}\left(\mathrm{NO}_{3}\right)_{3} \cdot 6 \mathrm{H}_{2} \mathrm{O}\right)$.

*All percentage data was arc-sine transformed prior to analysis.

Mean values followed by the same letter in the same column are not significantly different based on DMRT $(\mathrm{p}=0.05)$. $\mathrm{n}=90(10 \times 3 \times 3)$. Data was untransformed.

${ }^{1}$ Control with no sonication, but including TC PGRs: $0.1 \mathrm{mg} \cdot \mathrm{dm}^{-3} \mathrm{NAA}+0.1 \mathrm{mg} \cdot \mathrm{dm}^{-3} \mathrm{Kin}$.

${ }^{2}$ Control with no sonication and excluding PGRs.

${ }^{3} \mathrm{At}$ the beginning of each treatment, rootless shoots contained three full-grown shoots.

${ }^{4}$ Measured from the level of medium to the tallest leaf tip.

${ }^{5}$ Shoots were dabbed on dry tissue paper to remove in vitro flask moisture before weighing.

${ }^{6}$ Shoots were cut at the base from roots, wrapped in two layers of newspaper, labelled, and dry in a hot-air convection oven at $65^{\circ} \mathrm{C}$ for 1 week.

${ }^{7} \mathrm{SPAD}$ value is a measurement of the chlorophyll content; For IPM treatments, $\mathrm{n}=10$. 
Table 3. Root (derived from shoots derived from neo-PLBs) growth and development of hybrid Cymbidium Twilight Moon 'Day Light' in response to different lanthanoids at different concentrations 60 days in culture after transfer to PGR-free TC medium solidified with $2 \mathrm{~g} \cdot \mathrm{dm}^{-3}$ Gellan gum

\begin{tabular}{|c|c|c|c|c|}
\hline $\begin{array}{c}\text { Medium lanthanoids } \\
\text { in } \mathrm{mg} \cdot \mathrm{dm}^{-3} \\
\end{array}$ & $\begin{array}{c}\text { Number of } \\
\text { roots }^{3}\end{array}$ & $\begin{array}{c}\text { Root fresh } \\
\text { weight }(\mathrm{mg})^{4}\end{array}$ & $\begin{array}{l}\text { Root dry weight } \\
(\mathrm{mg})^{5}\end{array}$ & $\begin{array}{l}\text { Root : shoot ratio } \\
\text { FW/DW basis }{ }^{6}\end{array}$ \\
\hline TC + PGRs + no lanthanoids ${ }^{1}$ & $3.4 \mathrm{c}$ & $206 \mathrm{de}$ & $58 \mathrm{bc}$ & $0.159: 0.282$ \\
\hline TC - PGRs + no lanthanoids ${ }^{2}$ & $1.1 \mathrm{~d}$ & $85 \mathrm{f}$ & $27 \mathrm{~cd}$ & $0.184: 0.482$ \\
\hline $\mathrm{TC}-\mathrm{PGRs}+1 \mathrm{La}$ & $6.3 \mathrm{a}$ & $518 \mathrm{a}$ & $144 \mathrm{a}$ & $0.478: 0.889$ \\
\hline $\mathrm{TC}-\mathrm{PGRs}+2 \mathrm{La}$ & $5.4 \mathrm{ab}$ & $486 \mathrm{~b}$ & $106 \mathrm{~b}$ & $0.505: 0.807$ \\
\hline $\mathrm{TC}-\mathrm{PGRs}+4 \mathrm{La}$ & $3.6 \mathrm{c}$ & $241 \mathrm{~d}$ & $71 \mathrm{bc}$ & $0.342: 1.127$ \\
\hline $\mathrm{TC}-\mathrm{PGRs}+8 \mathrm{La}$ & IPM & IPM & IPM & IPM \\
\hline $\mathrm{TC}-\mathrm{PGRs}+1 \mathrm{Ce}$ & $5.8 \mathrm{ab}$ & $501 \mathrm{ab}$ & $121 \mathrm{ab}$ & $0.527: 1.330$ \\
\hline $\mathrm{TC}-\mathrm{PGRs}+2 \mathrm{Ce}$ & $4.1 \mathrm{bc}$ & $394 \mathrm{c}$ & $94 \mathrm{~b}$ & $0.569: 1.324$ \\
\hline $\mathrm{TC}-\mathrm{PGRs}+4 \mathrm{Ce}$ & IPM & IPM & IPM & IPM \\
\hline $\mathrm{TC}-\mathrm{PGRs}+8 \mathrm{Ce}$ & IPM & IPM & IPM & IPM \\
\hline $\mathrm{TC}-\mathrm{PGRs}+1 \mathrm{Nd}$ & $6.1 \mathrm{a}$ & $512 \mathrm{a}$ & $143 \mathrm{a}$ & $0.639: 1.959$ \\
\hline $\mathrm{TC}-\mathrm{PGRs}+2 \mathrm{Nd}$ & $3.1 \mathrm{c}$ & $191 \mathrm{de}$ & $63 \mathrm{bc}$ & $0.297: 1.125$ \\
\hline $\mathrm{TC}-\mathrm{PGRs}+4 \mathrm{Nd}$ & IPM & IPM & IPM & IPM \\
\hline $\mathrm{TC}-\mathrm{PGRs}+8 \mathrm{Nd}$ & IPM & IPM & IPM & IPM \\
\hline TC - PGRs + $1 \mathrm{Pr}$ & $5.6 \mathrm{ab}$ & $472 \mathrm{~b}$ & $101 \mathrm{~b}$ & $0.578: 1.295$ \\
\hline TC - PGRs +2 Pr & $4.4 \mathrm{bc}$ & $408 \mathrm{c}$ & $98 \mathrm{~b}$ & $0.672: 1.922$ \\
\hline $\mathrm{TC}-\mathrm{PGRs}+4 \mathrm{Pr}$ & IPM & IPM & IPM & IPM \\
\hline TC - PGRs $+8 \mathrm{Pr}$ & IPM & IPM & IPM & IPM \\
\hline $\mathrm{TC}-\mathrm{PGRs}+1 \mathrm{Sm}$ & $2.6 \mathrm{~cd}$ & $124 \mathrm{e}$ & $44 \mathrm{c}$ & $0.206: 0.733$ \\
\hline $\mathrm{TC}-\mathrm{PGRs}+2 \mathrm{Sm}$ & $0.9 \mathrm{~d}$ & $57 \mathrm{~g}$ & $16 \mathrm{~d}$ & $0.108: 0.333$ \\
\hline $\mathrm{TC}-\mathrm{PGRs}+4 \mathrm{Sm}$ & IPM & IPM & IPM & IPM \\
\hline $\mathrm{TC}-\mathrm{PGRs}+8 \mathrm{Sm}$ & IPM & IPM & IPM & IPM \\
\hline $\mathrm{TC}-\mathrm{PGRs}+1 \mathrm{Gd}$ & $4.9 \mathrm{~b}$ & $441 \mathrm{bc}$ & $102 \mathrm{~b}$ & $0.394: 0.570$ \\
\hline $\mathrm{TC}-\mathrm{PGRs}+2 \mathrm{Gd}$ & $3.7 \mathrm{c}$ & $237 \mathrm{~d}$ & $66 \mathrm{bc}$ & $0.248: 0.795$ \\
\hline $\mathrm{TC}-\mathrm{PGRs}+4 \mathrm{Gd}$ & IPM & IPM & IPM & IPM \\
\hline $\mathrm{TC}-\mathrm{PGRs}+8 \mathrm{Gd}$ & IPM & IPM & IPM & IPM \\
\hline
\end{tabular}

IPM = insufficient plant material (i.e., not enough roots since shoots from neo-PLBs did not form, or developed poorly); SON = sonication. Lanthanoid abbreviations: $\mathrm{La}=$ lanthanum (III) nitrate hexahydrate $\left(\mathrm{La}\left(\mathrm{NO}_{3}\right)_{3} \cdot 6 \mathrm{H}_{2} \mathrm{O}\right)$; $\mathrm{Ce}=$ cerium (III) nitrate hexahydrate $\left(\mathrm{Ce}\left(\mathrm{NO}_{3}\right)_{3} \cdot 6 \mathrm{H}_{2} \mathrm{O}\right) ; \mathrm{Nd}=$ neodymium (III) nitrate hexahydrate $\left(\mathrm{Nd}\left(\mathrm{NO}_{3}\right)_{3} \cdot 6 \mathrm{H}_{2} \mathrm{O}\right)$; $\mathrm{Pr}=$ praseodymium (III) nitrate hexahydrate $\left(\mathrm{Pr}\left(\mathrm{NO}_{3}\right)_{3} \cdot 6 \mathrm{H}_{2} \mathrm{O}\right) ; \mathrm{Sm}=$ samarium (III) nitrate hexahydrate $\left(\mathrm{Sm}\left(\mathrm{NO}_{3}\right)_{3} \cdot 6 \mathrm{H}_{2} \mathrm{O}\right)$; $\mathrm{Gd}=$ gadolinium $(\mathrm{III})$ nitrate hexahydrate $\left(\mathrm{Gd}\left(\mathrm{NO}_{3}\right)_{3} \cdot 6 \mathrm{H}_{2} \mathrm{O}\right)$

*All percentage data was arc-sine transformed prior to analysis.

Mean values followed by the same letter in the same column are not significantly different based on DMRT $(p=0.05)$. $\mathrm{n}=90(10 \times 3 \times 3)$. Data was untransformed.

PG = phloroglucinol; PLB = protocorm-like body; TC = Teixeira Cymbidium medium No. 1 (Teixeira da Silva 2012b).

${ }^{1}$ Control with no sonication, but including TC PGRs: $0.1 \mathrm{mg} \cdot \mathrm{dm}^{-3} \mathrm{NAA}+0.1 \mathrm{mg} \cdot \mathrm{dm}^{-3} \mathrm{Kin}$.

${ }^{2}$ Control with no sonication and excluding PGRs.

${ }^{3}$ Unlike shoots, no roots existed on shoots when first plated, thus number of roots $=$ new roots.

${ }^{4}$ Roots were dabbed on dry tissue paper to remove in vitro flask moisture before weighing; roots were cut off at the point adjoining them to the shoot.

${ }^{5}$ Roots were cut at the base from shoots, wrapped in two layers of newspaper, labelled, and dry in a hot-air convection oven at $65^{\circ} \mathrm{C}$ for 1 week.

${ }^{6} \mathrm{FW}=$ fresh weight; DW = dry weight; a ratio $<1.0$ = favoured shoot formation; a ratio $>1.0=$ favoured root formation. 

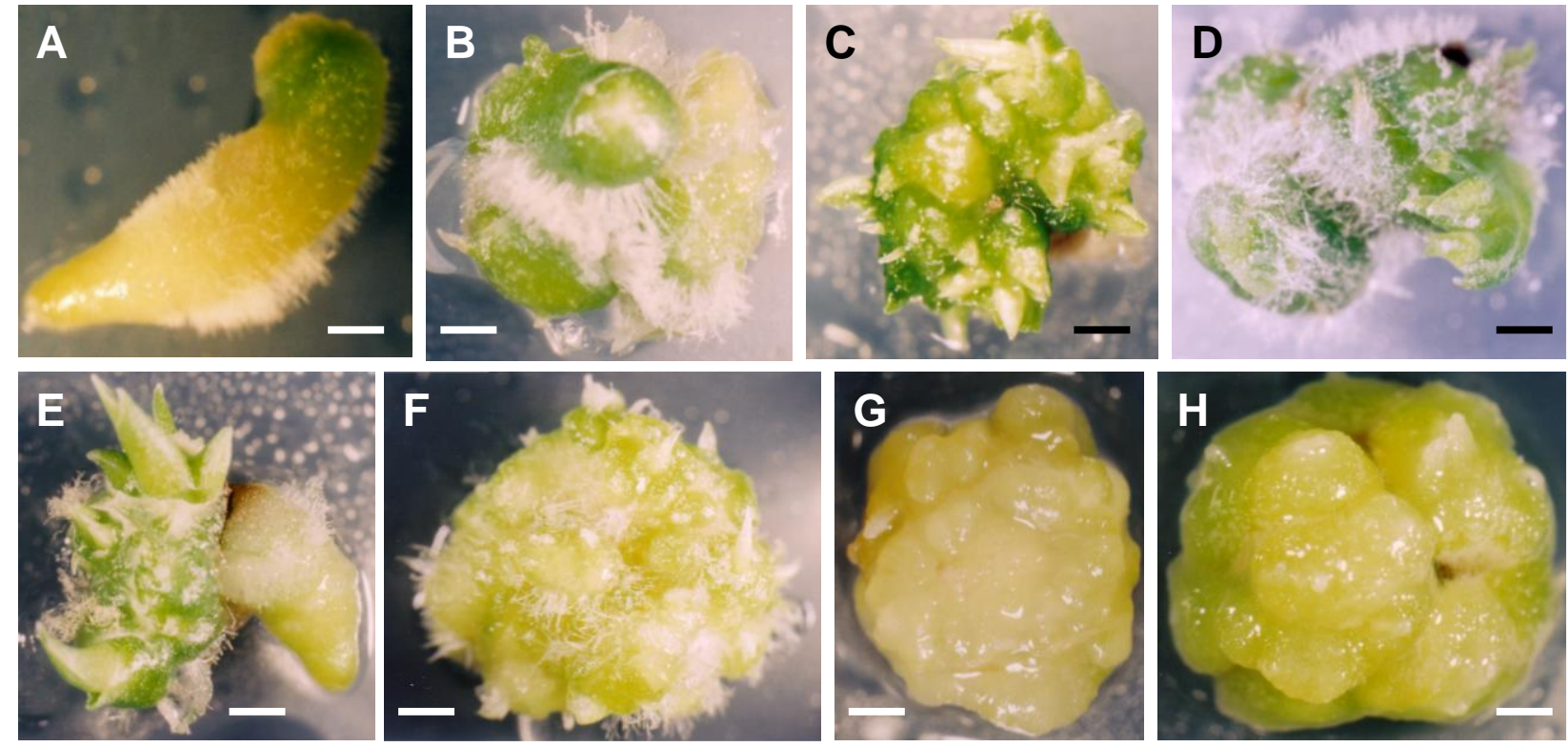

Fig. 1. Growth and development of hybrid Cymbidium Twilight Moon 'Day Light' neo-PLBs and roots in response to lanthanoids. (A) Swelling of excised root in the presence of $1 \mathrm{mg} \cdot \mathrm{dm}^{-3} \mathrm{La}\left(\mathrm{NO}_{3}\right)_{3} \cdot 6 \mathrm{H}_{2} \mathrm{O}$. (B) Control neoPLB (lanthanoid-free + PGRs) growing on solid basal TC (Teixeira da Silva 2012b) medium. Neo-PLB formation in the presence of (C) $2 \mathrm{mg} \cdot \mathrm{dm}^{-3} \mathrm{La}\left(\mathrm{NO}_{3}\right)_{3} \cdot 6 \mathrm{H}_{2} \mathrm{O}$, (D) $1 \mathrm{mg} \cdot \mathrm{dm}^{-3} \mathrm{Ce}\left(\mathrm{NO}_{3}\right)_{3} \cdot 6 \mathrm{H}_{2} \mathrm{O}$, (E) $1 \mathrm{mg} \cdot \mathrm{dm}^{-3}$ $\mathrm{Nd}\left(\mathrm{NO}_{3}\right)_{3} \cdot 6 \mathrm{H}_{2} \mathrm{O}$, (F) $1 \mathrm{mg} \cdot \mathrm{dm}^{-3} \operatorname{Pr}\left(\mathrm{NO}_{3}\right)_{3} \cdot 6 \mathrm{H}_{2} \mathrm{O}$, (G) $1 \mathrm{mg} \cdot \mathrm{dm}^{-3} \quad \mathrm{Sm}\left(\mathrm{NO}_{3}\right)_{3} \cdot 6 \mathrm{H}_{2} \mathrm{O}, \quad(\mathrm{H}) \quad 1 \mathrm{mg} \cdot \mathrm{dm}^{-3}$ $\mathrm{Gd}\left(\mathrm{NO}_{3}\right)_{3} \cdot 6 \mathrm{H}_{2} \mathrm{O}$. Bars $=1 \mathrm{~mm}$

The lanthanoids remain a relatively unexplored area of research in the biological sciences, particularly in the plant sciences, and most notably with only one other study related to in vitro culture. Even so, La has been used to monitor the movement of calcium in plant tissues (Liu \& Hasenstein 2005). Hu et al. (2004) showed the use of lanthanoids in agriculture. Spurred by a promising initial study on lanthanoids that showed that three of these rare earth metals could stimulate rooting in Dendrobium densiflorum (Luo et al. 2008), this study was born. In their study, Luo et al. found that after shoot induction in the presence of 6-benzyladenine, that the application of $2 \mathrm{mg} \cdot \mathrm{dm}^{-3} \mathrm{Nd}\left(\mathrm{NO}_{3}\right)_{3}$ could fortify root growth of plantlets, significantly more than commonly used auxins such as NAA, IAA and IBA. Plantlets could then be successfully acclimatised; $2.5 \mu \mathrm{M}$ IAA, when combined with $100 \mu \mathrm{M} \mathrm{La}^{3+}$, resulted in $96 \%$ rooting efficiency in Saussurea involucrata Kar. et Kir in vitro shoots (Guo et al. 2012). Peroxidase (POX) and superoxide dismutase activity increased in tissue of $\mathrm{La}^{3+}$-containing medium. Hong et al. (2005) noted that nitrate reductase (NR), glutamine synthetase and glutamate dehydrogenase activity in the roots of peach
[(Prunus persica (L.) Stokes)] in vitro plantlets increased significantly in response to $0.3 \mu \mathrm{mol} \cdot \mathrm{dm}^{-3}$ $\mathrm{CeCl}_{3}$. Song et al. (2003) also noted that 1.0$3.0 \mu \mathrm{mol} \cdot \mathrm{dm}^{-3} \mathrm{La}\left(\mathrm{NO}_{3}\right)_{3}$ in loquat (Eriobotrya japonica Lindi) in vitro rooting medium increased the rooting rate, root fresh weight, promoted root length and increase POX and NR activities.

Some rudimentary evidence for the mechanism of action exists. Lanthanoids increase the content of endogenous IAA by stimulating the synthesis of IAA precursor tryptophan and/or inhibiting the enzyme activity for IAA decomposition (Hu et al. 2004). Some relation with the stabilisation of the cytoskeleton of root cells also exists (Liu \& Hasenstein 2005) while mitochondrial metabolic activity is enhanced in the presence of La in rice (Oryza sativa L.) (Dai et al. 2008). Guo et al. (2012) supported this theory with the following mechanistic proposal 'La enhanced IAA-induced rooting by acting as a mild abiotic stress to stimulate POX and SOD activities in plant cells. Then, IAA reacted with oxygen and POX to form the ternary complex enzyme-IAA- $\mathrm{O}_{2}$ that dissociated into IAA radicals and $\mathrm{O}_{2}{ }^{-}$. Subsequently, IAA-induced $\mathrm{O}_{2}^{-}$readily 
converted to hydroxyl radical (HO-) via SOD-catalysed dismutation. Finally, cell wall loosening and cell elongation occurred as a consequence of HOdependent scission of wall components, leading to root growth'. The involvement of stress-inducible enzymes systems had already been shown in the roots of peach and loquat in vitro cultures grown in the presence of lanthanoids (Song et al. 2003; Hong et al. 2005) or in salt-stressed Vigna radiata (Shan \& Zhao 2014). Ruíz-Herrera et al. (2012) suggested that lanthanoids precipitate phosphate, creating $\mathrm{P}$ deficiency conditions in the growth medium, inhibiting primary root growth and increasing root hair and lateral root development. $\mathrm{CeCl}_{3}$ promoted nitrogen metabolism through the transformation of $\mathrm{NO}_{3}{ }^{-}$to $\mathrm{NH}_{4}{ }^{+}$in peach in vitro roots (Hong et al. 2005). Küpper et al. (2006) claim that photosynthesis and thus shoot/leaf growth could be affected by lanthanoids which can substitute the central $\mathrm{Mg}^{2+}$ ion of chlorophyll. Interestingly, in what appears to be support of this heavy-metal substitution of $\mathrm{Mg}^{2+}$ in chloroplasts, growth of Dryopteris erythrosora, a lanthanoid-accumulating fern species, was enhanced in response to La (Ozaki et al. 2000).

However, apart from five studies, including two on orchids, few other studies have examined the effects of lanthanoids on plant growth and development in vitro. Consequently, this area of research is at a nascent phase of development and many more trials would be required on more plant species, both edible, horticultural and agronomic, to assess the broad range of effects in vitro and under greenhouse and field trials. Key questions that still need to be answered: (A) what is the toxicity of lanthanoids, as assessed by toxicity assays? (B) What is the mechanism by which a plant takes up lanthanoids? (C) To what level and in what organelles and parts of the plant are lanthanoids accumulated, or used? The expense of these metallic chemical elements (between 60 and 200 US $\$ 25$ g) may make future research prohibitive or restricted, although only milligram amounts are in fact required.

\section{Acknowledgement}

The author thanks Prof. Michio Tanaka for research support and Dr. Judit Dobránszki for literature.

\section{REFERENCES}

Dai J., Zhang Y-Z., Liu Y. 2008. Microcalorimetric investigation on metabolic activity and effects of La (III) in mitochondria isolated from indica rice 9311. Biol. Trace Elem. Res. 121(1): 60-68. DOI: 10.1007/s12011-007-0062-4.

Diatloff E., Smith F.W., Asher C.J. 1995a. Rare earth elements and plant growth. II. Responses of corn and mungbean to low concentrations of lanthanum in dilute, continuously flowering nutrient solutions. J. Plant Nutr. 18: 1977-1989. DOI: 10.1080/01904169509365038.

Diatloff E., Smith F.W., Asher C.J. 1995b. Rare earth elements and plant growth. III. Responses of corn and mungbean to low concentrations of cerium in dilute, continuously flowering nutrient solutions. J. Plant Nutr. 18: 1991-2003. DOI: 10.1080/01904169509365039.

Guo B., Xu L.L., Guan Z.J., Wei Y.H. 2012. Effect of lanthanum on rooting of in vitro regenerated shoots of Saussurea involucrata Kar. et Kir. Biol Trace Elem Res. 147: 334-340. DOI: 10.1007/s12011-012-9326-8.

He Y.W., Loh C.S. 2000. Cerium and lanthanum promote floral initiation and reproductive growth of Arabidopsis thaliana. Plant Sci. 159: 117-124. DOI: 10.1016/S0168-9452(00)00338-1.

Hong F., Song W-P., Wan Z-G., Yu M-L., Yu J., Liu J-J., Sheng Y., Xi Q-H. 2005. Effect of La(III) on the growth and aging of root of loquat plantlet in vitro. Biol. Trace Elem. Res. 104: 185-191. DOI: 10.1385/BTER:104:2:185.

Hossain M.M., Kant R., Van P.T., Winarto B., Zeng S-J., Teixeira da Silva J.A. 2013. The application of biotechnology to orchids. Crit. Rev. Plant Sci. 32(2): 69-139. DOI: 10.1080/07352689.2012.715984.

Hu Z., Richter H., Sparovek G., Schnug E. 2004. Physiological and biochemical effects of rare earth elements on plants and their agricultural significance: a review. J. Plant Nutr. 27: 183-220. DOI: 10.1081/PLN-120027555.

Küpper H., Küpper F.C., Spiller M. 2006. [Heavy metal]-chlorophylls formed in vivo during heavy metal stress and degradation products formed during digestion, extraction and storage of plant material. In: Chlorophylls and Bacteriochlorophylls: Advances in Photosynthesis and Respiration, vol. 25, Springer, the Netherlands, pp. 6777. DOI: 10.1007/1-4020-4516-6_5. 
Liu M., Hasenstein K.H. 2005. $\mathrm{La}^{3+}$ uptake and its effect on the cytoskeleton in root protoplasts of Zea mays L. Planta 220: 658-666. DOI: 10.1007/s00425-004-1379-2.

Luo J.P., Wang Y., Zha X.Q., Huang L. 200. Micropropagation of Dendrobium densiflorum Lindl. ex Wall. through protocorm-like bodies: effects of plant growth regulators and lanthanoids. Plant Cell Tiss. Org. 93: 333-340. DOI: 10.1007/s11240-008-9381-1.

Ozaki T., Enomoto S., Minai Y., Ambe S., Ambe F., Makide Y. 2000. Beneficial effect of rare earth elements on the growth of Dryopteris erythrosora. J. Plant Physiol. 156: 330-334. DOI: 10.1016/S0176-1617(00)80070-X.

Ruíz-Herrera L.F., Sánchez-Calderón L., HerreraEstrella L., López-Bucio J. 2012. Rare earth elements lanthanum and gadolinium induce phosphate-deficiency responses in Arabidopsis thaliana seedlings. Plant Soil 353: 231-247. DOI: $10.1007 / \mathrm{s} 11104-011-1026-1$.

Shan C., Zhao X. 2014. Effects of lanthanum on the ascorbate and glutathione metabolism of Vigna radiata seedlings under salt stress. Biol. Plantarum (in press). DOI: 10.1007/s10535-014-0413-X.

Sharma S., Shahzad A., Teixeira da Silva J.A. 2013. Synseed technology - A complete synthesis. Biotechnol. Adv. 31: 186-207. DOI: 10.1016/j.biotechadv.2012.09.007.

Song W-P., Hong F., Wan Z-G., Zhou Y-Z., Gu F-G., Xu H-G., Yu M-L., Chang Y-H., Zhao M-Z., Su J-L. 2003. Effects of cerium on nitrogen metabolism of peach plantlet in vitro. Biol. Trace Elem. Res. 95: 259-268. DOI: 10.1385/BTER:95:3:259.

Teixeira da Silva J.A. 2012a. Production of synseed for hybrid Cymbidium using protocorm-like bodies. J. Fruit Ornam. Plant Res. 20(2): 135-146. DOI: 10.2478/v10290-012-0023-7.

Teixeira da Silva J.A. 2012b. New basal media for protocorm-like body and callus induction of hybrid Cymbidium. J. Fruit Ornam. Plant Res. 20(2): $127-$ 133. DOI: $10.2478 / \mathrm{v} 10290-012-0022-8$.

Teixeira da Silva J.A. 2013a. Orchids: advances in tissue culture, genetics, phytochemistry and transgenic biotechnology. Floriculture Ornamental Biotech. 7(1): 1-52.
Teixeira da Silva J.A. 2013b. The role of thin cell layers in regeneration and transformation in orchids. Plant Cell Tiss. Org. 113: 149-161. DOI: 10.1007/s11240-012-0274-y.

Teixeira da Silva J.A. 2013c. The phloroglucinol conundrum: increase in root growth of hybrid Cymbidium (Orchidaceae) with no toxic effect on protocorm-like body formation. Plant Tiss. Cult. Biotechnol. 23: 275-282.

Teixeira da Silva J.A., Chan M-T., Sanjaya, Chai M-L., Tanaka M. 2006a. Priming abiotic factors for optimal hybrid Cymbidium (Orchidaceae) PLB and callus induction, plantlet formation, and their subsequent cytogenetic stability analysis. Sci. Hortic. 109: 368-378. DOI: 10.1016/j.scienta.2006.05.016.

Teixeira da Silva J.A., Dobránszki J. 2013. How timing of sampling can affect the outcome of the quantitative assessment of plant organogenesis. Sci. Hortic. 159: 59-66. DOI: 10.1016/j.scienta.2013.05.001.

Teixeira da Silva J.A., Dobránszki J., Ross S. 2013. Phloroglucinol in plant tissue culture. In Vitro Cell. Dev. - Pl. 49: 1-16. DOI: 10.1007/s11627-013-9491-2.

Teixeira da Silva J.A., Norikane A., Tanaka M. 2007. Cymbidium: successful in vitro growth and subsequent acclimatization. Acta Hort. 748: 207-214.

Teixeira da Silva J.A., Singh N., Tanaka M. 2006 b. Priming biotic factors for optimal protocorm-like body and callus induction in hybrid Cymbidium (Orchidaceae), and assessment of cytogenetic stability in regenerated plantlets. Plant Cell Tiss. Org. 84: 119-128. DOI: 10.1007/s11240-005-9003-0.

Teixeira da Silva J.A., Tanaka M. 2006. Multiple regeneration pathways via thin cell layers in hybrid Cymbidium (Orchidaceae). J. Plant Growth Regul. 25: 203-210. DOI: 10.1007/s00344-005-0104-0.

Teixeira da Silva J.A., Yam T., Fukai S., Nayak N., Tanaka M. 2005. Establishment of optimum nutrient media for in vitro propagation of Cymbidium Sw. (Orchidaceae) using protocorm-like body segments. Propag. Ornam. Plants 5: 129-136.

Vacin E., Went F.W. 1949. Some pH changes in nutrient solutions. Bot. Gaz. 110: 605-613.

Wikipedia 2013. http://en.wikipedia.org/Lanthanoid 